\title{
EXPERIMENTAL RESULTS OF A NON-DESTRUCTIVE EMITTANCE MEASUREMENT DEVICE FOR H BEAMS*
}

\author{
C. Gabor ${ }^{\#}$, H. Klein, O. Meusel, U.Ratzinger, IAP, Frankfurt, Germany \\ J. Pozimski ${ }^{\dagger}$, Imperial College, London and RAL, Chilton, G.B.
}

\section{INTRODUCTION}

To increase the intensity and brightness of particle beams, a detailed knowledge of the transverse phasespace distribution (the emittance) is getting more and more important. Several well-known devices to measure precisely the emittance of a particle beam like the electric-sweep scanner [1] or the slit-grid [2] (also called slit-harp) instrument are widely used therefore. On the other hand these conventional destructive methods. suffers for such high power beam from the power density deposited on the surfaces like slits or pinhole plates. Additionally the beam is lost during the measurement and therefore an online supervision of the beam is impossible.

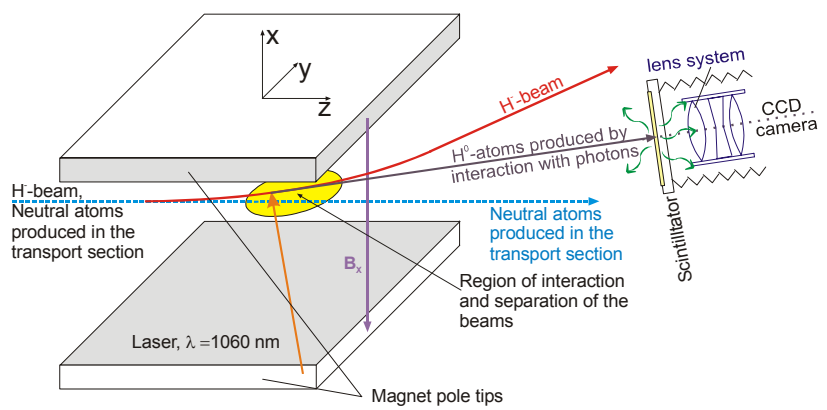

Figure 1: Principle of a photo detachment emittance measurement device (PD-EMI) using laser beam.

Furthermore for space charge compensated beam transport, like often used in magnetic low energy beam transport sections, the degree of space charge compensation is changing during the measurement due to the production of secondary particles on the surfaces.

Therefore the development of a non-destructive measurement method with marginal influence on the beam parameters is of high interest. For negatively charged particle beams (e.g. $\mathrm{H}^{-}$), the photodissociation (also called photo detachment) offers an elegant solution for this problem: A laser beam is used to selectively neutralize a segment of the particle beam (peak cross section $\sigma=4 * 10^{-17} \mathrm{~cm}^{2}$ at $\lambda_{\text {laser }}=830 \mathrm{~nm}$ ) [3]. The neutrals produced by photodetachment will then be separated from the negative ions using a beam-deflection element (e.g. a dipole magnet). By the use of a detector system with spatial resolution for the neutralized particles and for a know distance between neutralization and detector, the beam parameters (emittance) can be reconstructed. The proof of principle has been demonstrated at energies higher than $700 \mathrm{MeV}$ earlier $[4,5,6]$. Figure 1 shows the in

\footnotetext{
*Work supported by HPRI-CT-2001-50021

\#c.gabor@iap.uni-frankfurt.de

†j.pozimski@imperial.ac.uk
}

principle the used arrangement of laser and dipole to investigate the photodetachment technique. The laser scans through the $\mathrm{H}^{-}$beam within the diagnostic dipole. The magnetic field separates the neutrals produced by photodetachment from the negative ions and other neutrals produced by residual gas interaction earlier. This set up is very important to reduce the background at the szintillator and to improve the signal to noise ratio. In principle, this technique can be used to reconstruct the full 4-dimensional transversal phase space density distribution of the beam as a function of time, by altering the spatial and/ or temporal structure of the photon field interacting with the particle beam. A discussion in more detail and a comparison between a conventional slit-grid EMI and the PD-EMI is given in [7].

In this paper a short overview of the used test-bench, together with measurements using a slit-grid EMI and first results gained by photoneutralisation are presented.

\section{EXPERIMENTAL SETUP}

A general overview of the test-stand is shown in Figure 2. For the experiments a volume type ion source based on [8] with a single aperture accel-deccel triode extraction system was used. The ion source was operated in pulsed mode with typical pulse length of $400-600 \mu$ s and a repetition rate of $20-50 \mathrm{~Hz}$. A transverse magnetic filter field is installed in the flange of the plasma electrode to influence the electron energy distribution in the plasma and therefore enhance the $\mathrm{H}^{-}$production efficiency. The following vacuum chamber is subdivided by a thin wall with a pinhole of $\varnothing=10 \mathrm{~mm}$ for the beam, in two vacuum sections (differential pumping). Additionally permanent magnets on both sides of the pinhole are used to bend the simultaneously extracted electrons off axis and dump them on the wall. The measured $\mathrm{H}^{-}$current behind the dumping system is app. $0.9-1.5 \mathrm{~mA}$ at a beam energy of 5$6 \mathrm{keV}$.

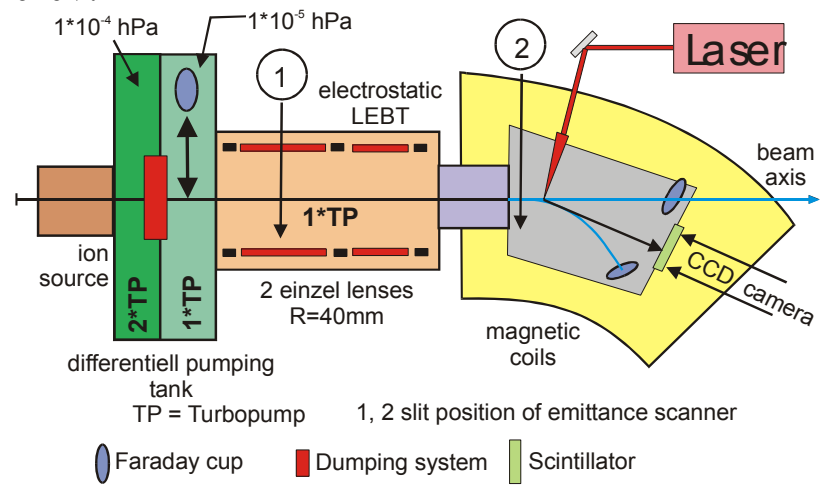

Figure 2: Schematic overview of the experimental set up. 
The electrostatic LEBT consists of two einzellenses with an inner diameter of $80 \mathrm{~mm}$. It is used to match the divergent $\mathrm{H}^{-}$beam delivered by the ion source convergent into the aperture of the diagnostic chamber. The dipole used to separate the neutrals produced by photodetachment from the negative ions is a commercial C-magnet with a path length of $305 \mathrm{~mm}$ and a deflection angle of $30^{\circ}$. The free aperture of the diagnostic chamber is $33.0 \mathrm{~mm}$. The chamber offers a wide range of vacuum ports for the ion and Laser beam (after a deflection of $10^{\circ}$ ), the scintillator and for beam current measurements of the ion beam. For the given geometry a beam radius up to $15 \mathrm{~mm}$ is possible for beam transport without particle losses. To determine proper injection into the dipole, to investigate beam transport through the LEBT and to compare the results with the gained data by photodetachment measurements, slit-grid emittance measurements have been performed at position 1 and 2 (see Figure 2).

A scintillating material will be used to convert the spatial distribution of the photo neutralized particles into photons. Therefore at the inner surface of the vacuum window with a diameter of $65 \mathrm{~mm}$ the scintillating material was evaporated or applied by sedimentation, at the outer side of the vacuum window a CCD camera is mounted. The vacuum window has the same angle like the neutrals $\left(10^{\circ}\right.$ with respect to the straightforward beam axis). Therefore the neutrals penetrate the scintillator perpendicular to the surface. The emitted light is then detected by a $\mathrm{LN}_{2}$ cooled, back illuminated slow scan CCD camera. A proton beam with a very low intensity $(<80 \mu \mathrm{A})$ at the energy range of $3-10 \mathrm{keV}$ was used to evaluate the photon efficiency of phosphorus P43 and P46 and $\mathrm{CdS}$ and to determine the light intensity measured by the CCD camera. Different material parameters of the scintillator were considered to improve the signal to noise ratio: The layer has to be thick enough to stop the beam completely (and convert all it's energy); on the other hand the optical thickness of the layer has to be small to achieve a high transmission of photons to the back side of the scintillator and to reduce the blurring of the image. To guarantee a homogeneous structure of the scintillator, at least 3 to 4 layers of phosphor grains are necessary. Bigger grains improve the quantum efficiency but reduce the resolution. The minimum average grain size available on market is $\sim 1 \mu \mathrm{m}$. TRIM [9] simulations have been performed to optimise all these parameters. The calculations show that for the presented experiments the optimal thickness of the layer has to be less than $5 \mu \mathrm{m}$. Therefore we chose a grain size of $1 \mu \mathrm{m}$ and a layer thickness of $4 \mu \mathrm{m}$. Different detectors where tested. P43 delivered a good signal to noise ratio with the disadvantage of reduced time resolution. In comparison for P46, the decay time is 3 times faster but the total light emission is $50 \%$ lower. Additionally a very thin Allayer $(20 \mathrm{~nm})$ at one side of the scintillator was used to increase the light emission into the CCD camera by reduction of photon losses and the reduction of background from secondary particles.
Table 1: Comparison of different scintillator materials. The efficiency is normalized to $10 \mathrm{keV}$ [Photons/electron].

\begin{tabular}{|c|c|c|c|c|}
\hline Type & Composition & $\begin{array}{c}\text { wave } \\
\text { length }\end{array}$ & $\begin{array}{c}\text { Decay time } \\
90-10 \%\end{array}$ & Efficiency \\
\hline P 43 & $\mathrm{Gd}_{2} \mathrm{O}_{2} \mathrm{~S}: \mathrm{Tb}$ & $545 \mathrm{~nm}$ & $1 \mathrm{~ms}$ & 330 \\
\hline P 46 & $\begin{array}{c}\mathrm{Y}_{3} \mathrm{Al}_{5} \mathrm{O}_{12}: \mathrm{Ce}, \\
\mathrm{Tb}\end{array}$ & $530 \mathrm{~nm}$ & $300 \mathrm{~ns}$ & 160 \\
\hline P 20 & $(\mathrm{Zn}, \mathrm{Cd}) \mathrm{S}: \mathrm{Ag}$ & $550 \mathrm{~nm}$ & $4 \mathrm{~ms}$ & 430 \\
\hline
\end{tabular}

\section{MEASUREMENT RESULTS}

A first check was performed to prove that the signals are produced by neutrals and not a measurement artefact (e.g. due to laser light, reflections induced by the ion beam). Typically measured distributions are shown in Figure 3. For lower magnetic fields both detected distributions move from the left to the right side of the scintillator: A lower B-field reduces the deflection angle of the $\mathrm{H}^{-}$beam before the negative ions are be neutralized by the laser $\left(\mathrm{H}^{-}, 5.5 \mathrm{keV}, \mathrm{I}_{\text {total }}=450 \mu \mathrm{A}\right.$, pulse duration $500 \mu \mathrm{s}$, dutycycle $=2 \%, \mathrm{P}_{\text {Laser, cw }}=29.3 \mathrm{~W}$ ).

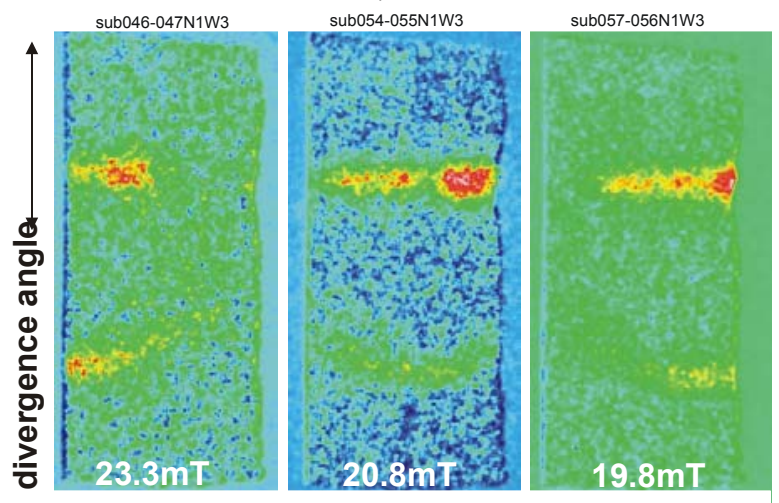

Figure 3: Distributions of neutrals as a function of the magnetic field, measured with a P43 scintillator.

The displacement of the produced neutrals on the detector in respect to the position of the laser is a function of the divergence angle of the ion beam at the interaction point. For the used experimental set up the divergence angle corresponds with the $y$-axis of the CCD image. The upper signals of the shown CCD images in Figure 3 are produced by the main part of the ion beam (small angle) and the lower distribution is caused by aberrations of the $\mathrm{H}^{-}$beam.

For a more detailed analyses of these distributions produced by photoneutralisation they have to be compared with a conventionally measured phase space distribution. One result of such a measurement behind the electrostatic lens system is shown in Figure 4 (left). The beam divides into the unfocused neutrals produced by residual gas interaction and the focused negative ion beam. Due to the large beam radius of $\sim 30 \mathrm{~mm}$ compared with a lens radius of $40 \mathrm{~mm}$ the shown aberrations are produced by the non-linear field components of the einzellenses. The plot on the right (gained by simulations) shows the emittance pattern at the interaction point. 


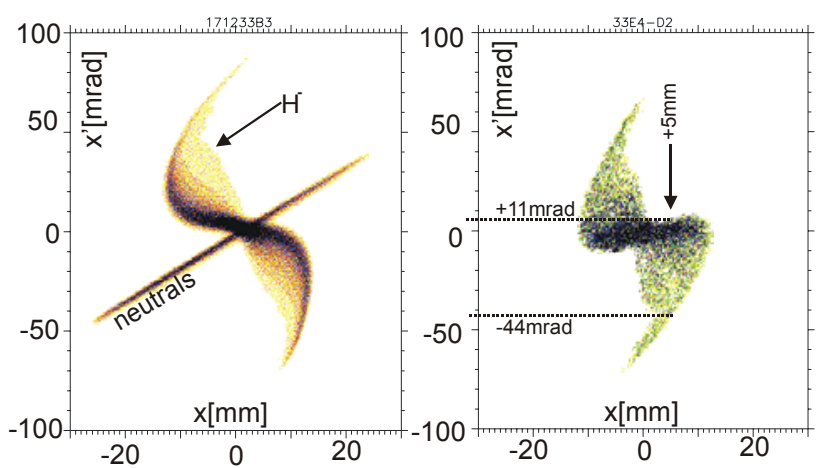

Figure 4: Left: Measured emittance plot behind the electrostatic lens system (lens $1=3.00 \mathrm{kV}$, lens $2=3.50 \mathrm{kV}$ ), measured with a slit-grid measurement instrument (negative ion beam $\varepsilon_{\mathrm{n}, \mathrm{rms}, 90 \%}=0,2857 \pi \mathrm{mm} \mathrm{mrad}$ ); Right: emittance, drifted of $108 \mathrm{~mm}$ up to the position of the laser (simulation without any influence of dipole field).

In Figure 5 one preliminary result of the photodetachment measurement (interaction point at the transversal position $\mathrm{x}=5 \mathrm{~mm}$ ) is shown in comparison with the results gained by conventional measurements together with simulations. The photodetachment process was simulated by using the conventionally measured distribution and using only the particles for $\mathrm{x}=5 . .6 \mathrm{~mm}$ for the further transport simulation to the scintillator (of course, without space charge). Even for the limited number of particles at high angles (especially for the aberations) used for this preliminary analyses the simulations show a good agreement of the divergence angle of app. 55-60mrad for both methods.

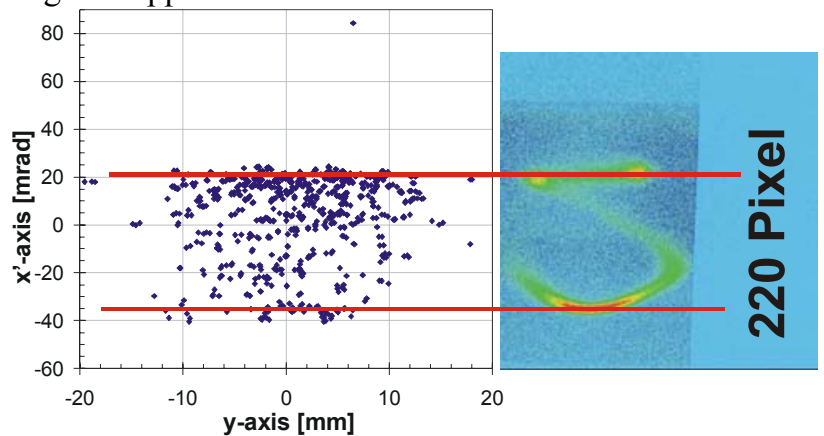

Figure 5: Comparison of the distributions in the transversal plane. The left diagram shows the simulated distribution, the right one shows the CCD image of neutralized $\mathrm{H}^{-}$in the same scale (220 pixel corresponds with $57 \mathrm{mrad}$ ).

In Figure 6 the integrated profiles along the y-axis reveal the same good agreement of both methods. Near the beam core, both profiles are nearly identical, while for larger angles the signal of the photodetachment is higher than the signal gained from simulations due to less particles at the drift calculation. Other explanations may be a nonlineraity of the luminance of the scintillator or an influence of the dipole magnet to the beam transport.

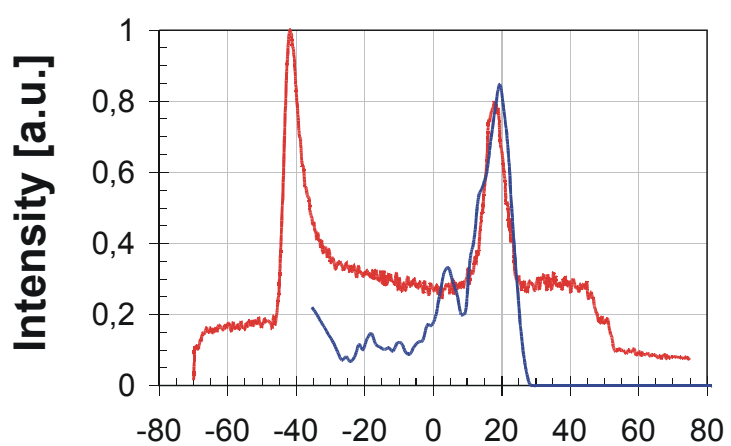

\section{$\mathbf{x}^{\prime}$-angle [mrad]}

Figure 6: Comparison of the $\mathrm{x}^{\prime}$-angle profile (integration along $y$-axis of Figure 5) of the photodetachment measurement (red) and the simulated slit-grid profile (blue). Both graphs have equal scaling and show (app.) the same divergence angle.

\section{CONCLUSION}

The neutrals produced by photodetachment can be detect with a scintillator and a CCD camera. The plot intensity versus beam angle (see Figure 6) generated by photodetachent corresponds with the profile extracted of the emittance pattern at the same position measured with a slit-grid EMI. Further investigations are planed with different szintillator materials, verify the homogenous build-up of luminance and time resolved measurements.

\section{REFERENCES}

[1] P.W.Allison, J.D.Sherman, D.B.Holtkamp, “An Emittance Scanner For Intense Low-Energy Ion Beams", PAC'83, Santa Fe, March 1983, http://www.jacow.org.

[2] A.Ehrlich, J.Galtz, P.Strehl, GSI Gesellschaft für Schwerionenphysik, Darmstadt 1974, GSI-PB-3-74.

[3] M.P.Ajmera and K.T.Chung, ,Photo detachment of Negative Hydrogen Ions“, Phys. Rev. A12, 1979.

[4] V.W.Yuan, et al., "Measurement of longitudinal phase space in an accelerated H-beam", Nucl. Instr. Meth., A329, (1993).

[5] R.C.Conolly, et al., "A transverse phase-space measurement technique for high-brightness $\mathrm{H}$ beams", Nucl. Instr. Meth., A312, (1992).

[6] R. Connolly, P. Cameron, J. Cupolo, M. Grau, M. Kesselman, C-J. Liaw, R. Sikora, "JACoW, Laser profile measurements of an $\mathrm{H}^{-}$beam", DIPAC'01, Grenoble, May 2001, p.114, http://www.jacow.org.

[7] C.Gabor, et al. "Status report of the Frankfurt $\mathrm{H}^{-}$test LEBT including a non-desturctive emittance measurement device", Proc. of the $9^{\text {th }}$ Intern. Symp. on Negative Ion Sources and Beams, Saclay, France, May 2002, AIP Conference Proc., 639, p.121.

[8] A.Jakob, H.Klein, A.Lakatos, J.Pozimski, "Extraction and Low Energy Transport of Negative Ions", Proc. $6^{\text {th }}$ EPAC, Stockholm, 1998.

[9] http://www.srim.org. 